Article

\title{
Solid-Phase Synthesis of Cellulose Acetate Butyrate as Microsphere Wall Materials for Sustained Release of Emamectin Benzoate
}

\author{
Aimin Huang ${ }^{1,3}$, Xuanhai $\mathrm{Li}^{1}{ }^{1}$, Xingtang Liang ${ }^{2, *}$, Yanjuan Zhang ${ }^{1}{ }^{\circledR}, \mathrm{Huayu} \mathrm{Hu}^{1}{ }^{1}$, \\ Yanzhen Yin ${ }^{2}$ and Zuqiang Huang $1, * \mathbb{D}$ \\ 1 School of Chemistry and Chemical Engineering, Guangxi University, Nanning 530004, China; \\ ham2003cn@163.com (A.H.); xuanhli@gxu.edu.cn (X.L.); zhangyj@gxu.edu.cn (Y.Z.); \\ yuhuahu@163.com (H.H.) \\ 2 Qinzhou Key Laboratory of Biowaste Resources for Selenium-Enriched Functional Utilization, \\ College of Petroleum and Chemical Engineering, Qinzhou University, Qinzhou 535011, China; \\ yinyanzhen2018@163.com \\ 3 Medical College of Guangxi University, Nanning 530004, China \\ * $\quad$ Correspondence: qzxylxt@163.com (X.L.); huangzq@gxu.edu.cn (Z.H.); Tel.: +86-771-3233728 (Z.H.)
}

Received: 13 November 2018; Accepted: 10 December 2018; Published: 13 December 2018

\begin{abstract}
Emamectin benzoate (EB), a widely used pesticide, is prone to decomposition by ultraviolet light and suffers from the corresponding loss of efficacy. The timed release of EB based on microspheres is one of the effective methods to solve this issue. As a non-toxic cellulose ester, cellulose acetate butyrate $(\mathrm{CAB})$ is regarded as one of the best wall-forming materials for microcapsules with a good controlled release performance. Herein, two methods-mechanical activation (MA) technology and a conventional liquid phase (LP) method-were employed to synthesize different CABs, namely CAB-MA and CAB-LP, respectively. The molecular structure, rheological property, and thermal stability of these CABs were investigated. The two CABs were used to prepare microspheres for the loading and release of EB via an o/w (oil-in-water) solvent evaporation method. Moreover, the performances such as drug loading, drug entrapment, and anti-photolysis of the drug for these microspheres were studied. The results showed that both $C A B s$ were available as wall materials for loading and releasing EB. Compared with CAB-LP, CAB-MA presented a lower molecular weight and a narrower molecular weight distribution. Moreover, the MA method endowed the $C A B$ with more ester substituent groups and less crystalline structure in comparison to the LP method, which had benefits including pelletizing and drug loading.
\end{abstract}

Keywords: cellulose acetate butyrate; solid-phase synthesis; mechanical activation; microspheres; emamectin benzoate; release

\section{Introduction}

Due to advantages such as high efficiency, low toxicity, low residue, and low pollution, emamectin benzoate (EB) has been widely used as a biopesticide through the forms of emulsion, microemulsion, suspension, and granules dispersed in water [1]. However, EB can be readily degraded by ultraviolet light, which degrades its efficacy and limits the application of EB [2]. The development of the timed release of EB by embedding it into microcapsules or microspheres composed of polymers is one of the promising methods to solve this issue. Generally, at the initial stage of the utilization, drugs distributed on the surface of microspheres are directly diffused into the environment, resulting in a fast release at the beginning stage. Then, the active ingredient that is incorporated into the carrier is released slowly via the inner channels or the decomposition of the microsphere component [3]. This 
release mechanism presents several advantages in the application of pesticide. First, the slow release of effective components wrapped in the polymer carrier prolongs the period of validity and improves the utilization rate. Meanwhile, the contact toxicity and inhalation toxicity of toxic pesticides toward crops, beneficial insects, and human beings will be reduced. Furthermore, the internal effective components will be protected by the carrier materials, which decrease the decomposition of drugs by environmental factors including light and microorganisms, and therefore boost its utilization [2]. For the wall materials, those with biocompatibility and biodegradation such as chitosan, starch, and polylactic acid have attracted widespread attention [4-6]. As the most abundant green renewable resource, cellulose is a promising candidate as a wall-forming material for microspheres. However, highly crystalline cellulose has poor solubility, which limits its applicability [7]. The cellulose ester, a cellulose derivative prepared via the esterification of cellulose, has advantages including high solubility, good film-forming ability, high glass-transition temperature, and biodegradability. Due to such advantages, it has attracted more and more attention as a microcapsule material [8]. Cellulose acetate butyrate (CAB) is a typical cellulose ester that is prepared by the simultaneous esterification with acetyl and butyryl groups [9]. With excellent UV resistance, good compatibility, and good film-forming ability, CAB has been regarded as one of the most widely used cellulose esters for microsphere wall materials [10,11]. Generally, the drug encapsulation and controlled release of $\mathrm{CAB}$ microcapsules is affected by the physicochemical properties of a polymer such as its crystalline structure and viscosity, which is determined by the degree of substitution and molecular weight [12]. The CAB with low viscosity would be favorable for the pelletization and encapsulation of drugs. However, the physicochemical properties of $C A B$ are significantly influenced by the preparation methodology [12].

Recently, our research group has developed a new green method for preparing cellulose derivatives by mechanical activation-assisted solid-phase reaction (MASPR) technology without using organic co-reagents and solvents [13-16]. In the MASPR process, the mechanical activation (MA) fractures the crystalline structure of cellulose to decrease the particle size and make a new surface with highly active hydroxyl groups. Meanwhile, the MA promotes the penetration of other reactants into the inside of the cellulose. All of these significantly enhance the efficiency of cellulose modification. Furthermore, without any use of additional solvents, this process simplifies the operation and fully exploits the mechanical energy for the reaction.

Based on these considerations, in the current work, we prepared CAB by the MA technology and the conventional liquid phase (LP) method for microcapsule materials. The physicochemical properties such as the molecular structure, crystalline structure, and rheological property for the two kinds of $\mathrm{CAB}$ (CAB-MA and CAB-LP) were characterized to study their differences. Then, these two types of $C A B$ s were used as wall materials to prepare $C A B$ microspheres containing emamectin benzoate (EB/CAB microspheres) through the solvent evaporation method. The drug loading, drug release, morphology, and the photodegradation of the $\mathrm{EB} / \mathrm{CAB}$ microspheres were systematically studied. This work provided a promising method to prepare $\mathrm{CAB}$ as wall materials of microsphere for the slow release of EB.

\section{Materials and Methods}

\subsection{Materials}

Microcrystalline cellulose (MCC) with a degree of polymerization of about 200 was obtained from Shanghai Yuanye Bio-Technology Co., Ltd. (Shanghai, China). Acetic anhydride, butyric acid, acetone, dichloromethane, absolute alcohol, methyl alcohol, and acetonitrile were supplied by Aladdin Industrial Co., Ltd. (Shanghai, China). Acetonitrile was chromatographically pure, and all of the other reagents were of analytical grade and used without further purification. 


\subsection{Preparation of $C A B-M A$ and $C A B-L P$}

CAB-MA prepared by the MA method was performed in a customized stirring ball mill [17]. Typically, $10.0 \mathrm{~g}$ of MCC mixed with butyric acid, acetic anhydride, and sulfuric acid catalyst (molar ratio of anhydroglucose unit (AGU) of MCC:butyric acid:acetic anhydride:sulfuric acid = 1:6:3:0.06) was poured into a 1200-mL jacketed stainless steel chamber containing $500 \mathrm{~mL}$ of zirconium oxide milling balls $(\Phi=5 \mathrm{~mm})$. The mixture was subjected to the milling at $350 \mathrm{rpm}$ for one hour at a temperature of $75{ }^{\circ} \mathrm{C}$, which was controlled by a circulation of thermostatic water in the chamber jacket. The esterification reaction was simultaneously conducted in the chamber. Then, the resulting sample was separated from the milling balls and treated by the washing-filtration process with water and absolute alcohol, respectively. This process was repeated for several cycles until the filtrate was neutral to remove unreacted reagents. The filter cake was collected and vacuum dried at $55^{\circ} \mathrm{C}$ for two days to obtain the CAB-MA.

CAB-LP was prepared according to the literature with some modification [18]. Briefly, $10.0 \mathrm{~g}$ of MCC was firstly pretreated via immersion in a 120-mL mixture of acetic acid and acetic anhydride with the volume ratio of 5:1 for $12 \mathrm{~h}$ at ambient temperature. After filtration, the pretreated MCC, butyric acid, acetic anhydride, and sulfuric acid catalyst with the same molar ratio to the above-mentioned method were magnetically stirred for one hour at $75^{\circ} \mathrm{C}$ for esterification. After washing and drying in a similar manner as described above, the CAB-LP was acquired.

\subsection{Characterizations of $C A B$}

The ${ }^{13} \mathrm{C}$ nuclear magnetic resonance (NMR) spectra of CABs were recorded on a Bruker Advance HD III $600 \mathrm{MHz}$ spectrometer (Flanders, Switzerland) at room temperature, by using deuterated chloroform $\left(\mathrm{CDCl}_{3}\right)$ and tetramethylsilane (TMS) as the solvent and internal standard, respectively. Degree of substitution (DS) values for CABs were determined according to Tezuka and Tsuchiya's method based on the ${ }^{13} \mathrm{C}$ NMR spectra of cellulose esters [19]. X-ray diffraction (XRD) experiments for MCC and CABs were conducted on an Ultima IV diffractometer (Tokyo, Japan) with a Cu K $\alpha$ $\mathrm{X}$-ray beam at $40 \mathrm{kV}$ and $24 \mathrm{~mA}$. The relative molecular weight of CAB was determined by a LC-20AT high-performance liquid gel chromatograph (SHIMAZU, Japan) with Shodex GPC KF-804L C004091 HPLC column at $25^{\circ} \mathrm{C}$. Polystyrene and tetrahydrofuran were used as the molecular weight marker and mobile phase, respectively. The rheological properties of $\mathrm{CAB}$ acetone solutions were measured on an Anton Paar MCR 302 dynamic rotational rheometer (Graz, Austria), using the Rheo Plus software. All of the measurements were carried out in the CC27 concentric cylinder geometry. Thermogravimetric analyses were executed on a TGA Q50 thermogravimetric analyzer (Wilmington, DE, USA).

\subsection{Preparation of $E B / C A B$ Microspheres}

$\mathrm{EB} / \mathrm{CAB}$ microcapsules were prepared with an $\mathrm{o} / \mathrm{w}$ solvent evaporation method within an open cylindrical reactor $(\mathrm{h}=155 \mathrm{~mm}, \mathrm{~d}=112 \mathrm{~mm}$ ) combined with a high-power thermostatic magnetic stirrer. Typically, $200 \mathrm{mg}$ of CAB and $100 \mathrm{mg}$ of EB were added into five $\mathrm{mL}$ of chloroform, and then stirred for two minutes. Afterward, the obtained viscous liquid was added dropwise into the polyvinyl alcohol solution ( $300 \mathrm{~mL}, 1 \% w / v$ and $88 \%$ hydrolyzed) under an 800 -rpm stirring. This mixture was continuously stirred for $20 \mathrm{~min}$ at room temperature. After that, the temperature of the system was raised up to $55^{\circ} \mathrm{C}$ and another one hour of stirring ( $500 \mathrm{rpm}$ ) was conducted. Finally, the $\mathrm{EB} / \mathrm{CAB}$ complex microspheres were obtained after vacuum filtration, washed with deionized water, and vacuum dried at $50^{\circ} \mathrm{C}$. Blank $\mathrm{CAB}$ microspheres were also attained by the same method without adding EB. 


\subsection{Characterizations of $E B / C A B$ Microspheres}

The particle size and size distribution of EB/CAB microspheres were determined using a BT-9300st laser particle size distribution instrument (Dandong, China). Morphologies for $\mathrm{EB} / \mathrm{CAB}$ microspheres were observed in an S-3400N scanning electron microscope (SEM, HITACHI, Tokyo, Japan).

\subsection{Characterizations of the Drug Loading of EB/CAB Microspheres}

First, $40 \mathrm{mg}$ of EB/CAB microspheres were immersed in $100 \mathrm{~mL}$ of methyl alcohol, and then ultrasonically treated for 20 min to completely discharge the embedded EB from the microspheres. The EB concentration of the methyl alcohol solution was measured by a TCC-300 CD high-performance liquid chromatography (HPLC) system equipped with a C18 column (Waters Sunfire). A mixture of methanol and acetonitrile with a volume ratio $=55: 45$ was used as the mobile phase. The mass fraction of $\mathrm{EB}$ in $\mathrm{EB} / \mathrm{CAB}$ microspheres, drug-loading rate, and entrapment rate were calculated according to the literature [20].

\subsection{Release Studies of $E B$ from $E B / C A B$ Microspheres}

EB release tests from the microspheres were conducted in $0.05 \mathrm{M}$ of phosphate buffer solution (PBS) (PH 7.0, 20\% methanol) by the membrane diffusion method. First, $150 \mathrm{mg}$ of EB/CAB microspheres were placed in a dialysis bag and put into a conical flask containing $120 \mathrm{~mL}$ of PBS. The conical flask was placed in an oscillator with $25^{\circ} \mathrm{C}$ and subjected to a continuous shaking at $120 \mathrm{rpm}$. Then, two $\mathrm{mL}$ of release medium were withdrawn at 24-h intervals for determining the EB content. The changes of the morphology of $\mathrm{CAB}$ microspheres during the release process were recorded by an optical microscope.

\subsection{Experiments for EB Photodegradation}

The photodegradation of EB was conducted under sunlight. Briefly, about $50.0 \mathrm{mg}$ of EB/CAB microspheres were placed in a Petri dish containing $10 \mathrm{~mL}$ of water and exposed to sunlight for photodegradation. For the control sample, the same system containing $50.0 \mathrm{mg}$ of EB/CAB and $10 \mathrm{~mL}$ of water was placed in a dark room. After maintaining for one hour, two hours, four hours, eight hours and 10 hours, both samples, including the exposed one and the unexposed one, were treated by ultrasonication in methanol to completely release EB. Then, the content of EB was detected. The content of $\mathrm{EB}$ for the exposed $\mathrm{EB} / \mathrm{CAB}$ was labeled as $m_{\mathrm{e}}$, while the control one was $m_{\mathrm{c}}$. The photodegradation rate $\left(P_{\mathrm{r}}\right)$ of EB was calculated according to the equation $\left(P_{\mathrm{r}}=100 \%\left(m_{\mathrm{c}}-m_{\mathrm{e}}\right) / m_{\mathrm{c}}\right)$. The EB was also conducted in a similar manner as the above-mentioned process for determining the $P_{\mathrm{r}}$.

\section{Results and Discussion}

\subsection{Distribution of Substituted Groups in CABs with Different Synthesis Methods}

The ${ }^{13} \mathrm{C}$ spectra of CAB-MA and CAB-LP are shown in Figure 1. Two triplets at the range of 169-174 ppm, assigned to the acetyl carbonyl and the butyryl carbonyl, respectively, appeared in the spectra of both CABs, indicating the successful preparation of cellulose mixed esters via both approaches [21]. For the acetyl carbonyl triplet, the peaks at $169.3 \mathrm{ppm}, 169.8 \mathrm{ppm}$, and $170.3 \mathrm{ppm}$ are attributed to the carbonyl signal of the acetate group in the two, three, and six positions of the AGU. While the peaks at $171.9 \mathrm{ppm}, 172.4 \mathrm{ppm}$, and $172.9 \mathrm{ppm}$ in the butyryl carbonyl triplet are assigned to the carbonyl signal of the butyrate-substituted group in the two, three, and six positions. According to Tezuka and Tsuchiya's method, the DS values for every position and the total DS were calculated and listed in Table 1. During the process of the MA method, all of the hydroxyl groups in the cellulose were activated by ball milling to react with the esterifying agents [18]. The primary hydroxyl of $\mathrm{C} 6$ at the AGU, with a higher activity in comparison to the secondary hydroxyl at $\mathrm{C} 3$ and $\mathrm{C} 2$, resulted in a significantly greater degree of substitution at $\mathrm{C} 6$ than at $\mathrm{C} 3$ and $\mathrm{C} 2$. However, in the LP process, the substituent in C6 was close to that of $C 3$ and $C 2$, namely, the esterification of AGU 
by the LP process was more homogeneous than that of the MA method. This would be due to the solvation effect of the acetic acid and acetic anhydride in the pretreatment process, which protected the C6-OH group [22]. In addition, the total DS of CAB-MA $\left(\mathrm{DS}_{\text {total }}=2.73\right)$ was greater than that of $\mathrm{CAB}-\mathrm{LP}\left(\mathrm{DS}_{\text {total }}=2.53\right)$, indicating the advantages of the MA method for preparing CAB.
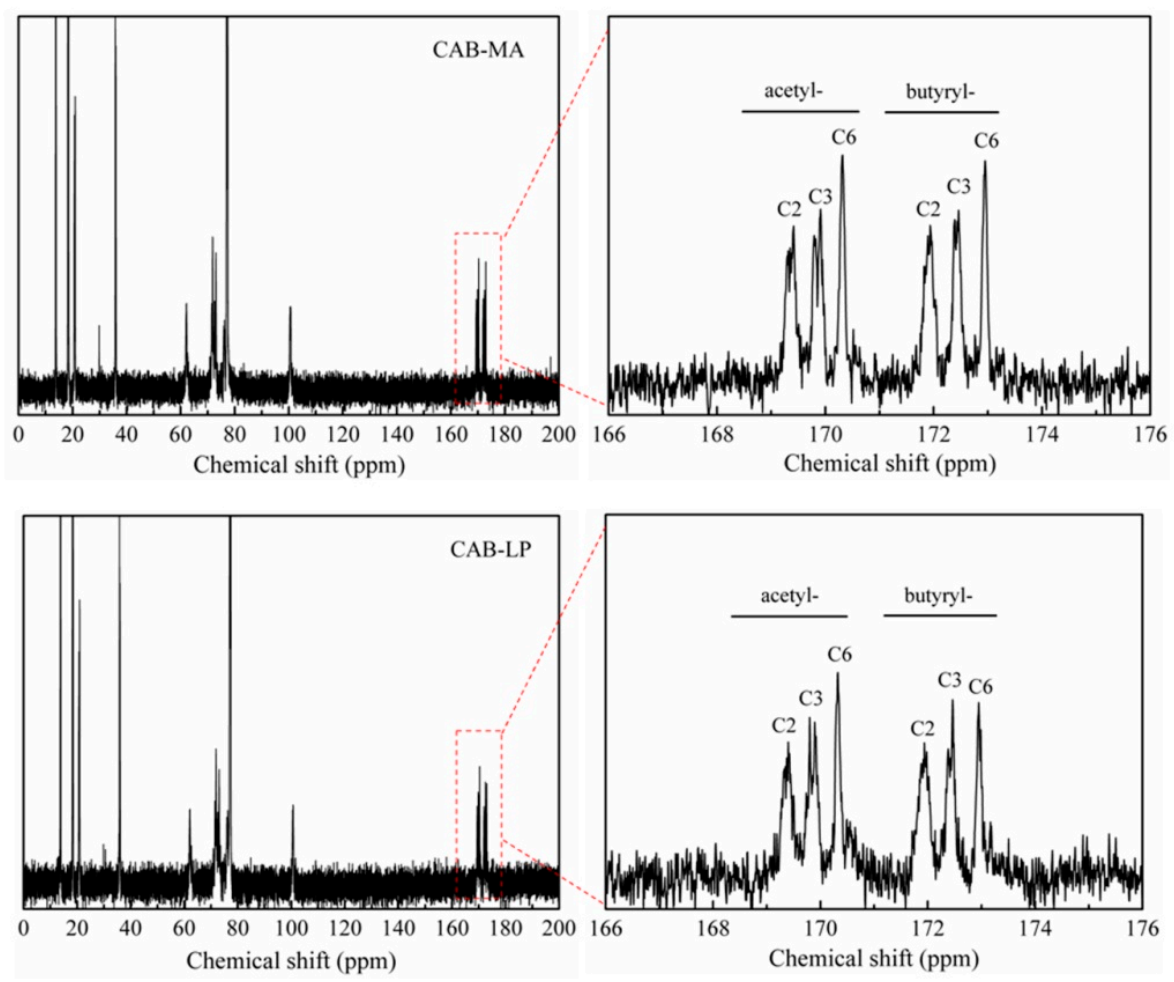

Figure 1. ${ }^{13} \mathrm{C}$ NMR spectra of cellulose acetate butyrate by mechanical activation (CAB-MA) and liquid phase methods (CAB-LP).

Table 1. The distribution of substituted groups in the anhydroglucose unit (AGU) of CAB-MA and CAB-LP. DS: degree of substitution.

\begin{tabular}{|c|c|c|c|c|c|c|c|c|c|}
\hline & \multicolumn{4}{|c|}{ DS of Butyryl } & \multicolumn{4}{|c|}{ DS of Acetyl } & \multirow{2}{*}{$\mathrm{DS}_{\text {total }}$} \\
\hline & $\mathrm{C}_{6}$ & $\mathrm{C}_{3}$ & $\mathrm{C}_{2}$ & $\mathrm{DS}_{\mathrm{b}}$ & $\mathrm{C}_{6}$ & $\mathrm{C}_{3}$ & $\mathrm{C}_{2}$ & $\mathrm{DS}_{\mathrm{a}}$ & \\
\hline CAB-LP & 0.57 & 0.59 & 0.44 & 1.62 & 0.37 & 0.29 & 0.24 & 0.91 & 2.53 \\
\hline CAB-MA & 0.69 & 0.52 & 0.47 & 1.67 & 0.43 & 0.33 & 0.29 & 1.05 & 2.73 \\
\hline
\end{tabular}

\subsection{Effect of Synthesis Method on Crystal Structure of $C A B$}

As wall materials, the crystallinity directly affects its mechanical strength and sealing property [23]. The higher the degree of crystallinity, the smaller the distance between molecules, which increases the intermolecular cohesive force, and therefore increases the rigidity of the polymer chains. At the same time, the molecular chain within the polymer with a high degree of crystallinity will be bound to a certain extent, which hinders the movement of the molecular chain segment and results in a fragile microcapsule. Moreover, the crystal components are of high density and low porosity, which obstructs the diffusion of the drug from the inside of the microcapsule to the external environment [24]. Conversely, the polymer with low crystallinity promotes the movement of the molecular chains and enhances the flexibility. Therefore, these microcapsules prepared with a low crystallinity polymer exhibit a suitable release of drug [25].

The XRD patterns of MCC, CAB-MA, and CAB-LP are shown in Figure 2. The diffraction pattern of MCC revealed the crystalline planes of (101), (10-1), (002), and (040) at about $14.9^{\circ}, 16.3^{\circ}, 22.5^{\circ}$, 
and $34.8^{\circ}$, respectively, confirming the form of native cellulose I [26]. After an esterification reaction, the intensity of the relevant peaks decreased significantly, indicating that this esterification modification notably destroyed the crystal structure. The crystallinity indices (CrI, \%) and the crystallite sizes $\left(\mathrm{D}_{002}\right.$, $\mathrm{nm}$ ) for these samples were calculated according to equations (1) and (2), respectively [27].

$$
\begin{gathered}
\mathrm{CrI}=100\left(I_{002}-I_{\mathrm{am}}\right) / I_{\mathrm{am}} \\
\mathrm{D}_{002}=k \lambda(\beta \cos \theta)
\end{gathered}
$$

where, $I_{002}$ and $I_{\mathrm{am}}$ are the intensity of the (002) plane and the diffraction intensity of amorphous region at $18.0^{\circ}$, respectively; and $k, \lambda, \beta$, and $\theta$ represent the Scherrer constant (0.94), the wavelength of X-ray $(0.154 \mathrm{~nm})$, the full-width at half-maximum (002) peak, and the diffraction angle of the (002) plane, respectively. As shown in Table 2, the CrI for CAB-MA is less than that of CAB-LP, while the $\mathrm{D}_{002}$ for CAB-MA is greater than that of CAB-LP, which is close to the $\mathrm{D}_{002}$ of MCC. These results would be attributed to the esterification reaction in the LP process occurring in the amorphous region and on the surface of the crystalline zone, which gradually destroys the crystal structure, but without influencing the crystallite size. However, in the MA process, MA not only induced the esterification reaction, but also broke and refined the cellulose particles, which enlarged the interplanar spacing.

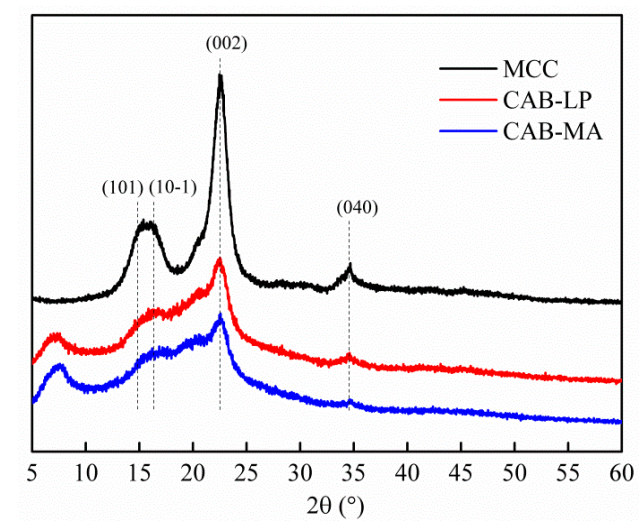

Figure 2. Typical XRD patterns of microcrystalline cellulose (MCC), CAB-LP, and CAB-MA.

Table 2. Values of CrI and $D_{002}$ for MCC, CAB-LP, and CAB-MA $(n=3)$.

\begin{tabular}{cccc}
\hline & MCC & CAB-LP & CAB-MA \\
\hline CrI $(\%)$ & $94.9 \pm 1.9$ & $44.7 \pm 2.8$ & $21.7 \pm 5.2$ \\
$D_{002}(\mathrm{~nm})$ & $3.5 \pm 0.4$ & $3.7 \pm 0.1$ & $4.2 \pm 0.4$ \\
\hline
\end{tabular}

\subsection{Effect of Synthesis Method on Molecular Structure of $C A B$}

The number-average molecular weight $\left(M_{n}\right)$, weight average molecular weight $\left(M_{\mathrm{w}}\right)$, and molecular weight distribution (D) for both CAB-MA and CAB-LP were determined by the gel permeation chromatography (GPC) method. As shown in Figure 3, the $M_{\mathrm{n}}, M_{\mathrm{w}}$, and D of CAB-LP are significantly greater than that of $\mathrm{CAB}-\mathrm{MA}$, indicating that the cellulose molecules and the $\mathrm{CAB}$ molecules are fractured under the repeated actions such as shear, impact, friction, and impact during the process of mechanical activation. With a low molecular weight and a narrow molecular weight distribution width, CAB-MA would be more soluble and dispersive than CAB-LP. This was proved by the rheological experiment, as shown in Figure 4. The energy storage modulus $\left(G^{\prime}\right)$ and the loss modulus $\left(G^{\prime \prime}\right)$ are used to characterize the elastic properties and the viscosity of a polymer solution, respectively. The $G^{\prime}$ for CAB-MA was greater than the $G^{\prime \prime}$ in the studied frequency range, indicating the characteristic of solid elasticity. However, the $G^{\prime}$ for CAB-LP was smaller than the $G^{\prime \prime}$ in the same frequency range, confirming the typical liquid viscosity behavior. These results showed that the 
CAB-MA, with a greater DS, a lower molecular weight, and a narrower molecular weight distribution in comparison to the CAB-LP exhibited a stronger tendency to assemble. This is suitable as a wall material with good flexibility and mechanical strength for the efficient encapsulation of drug [28].

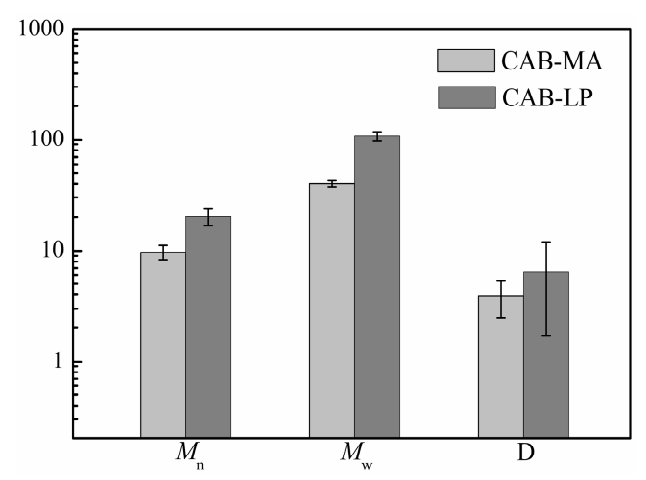

Figure 3. Relative molecular weight and molecular weight distribution of CAB-MA and CAB-LP $(n=3)$.

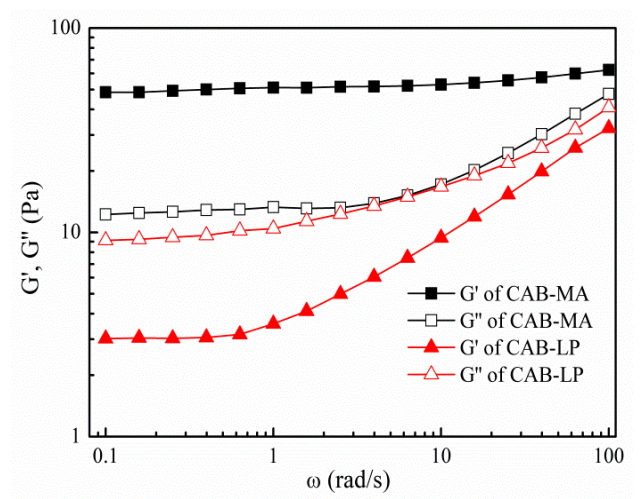

Figure 4. The dynamic frequency scanning curves of the CAB-MA and CAB-LP.

The thermogravimetric analysis (TGA) and derivative thermogravimetry (DTG) curves for MCC, CAB-MA, and CAB-LP are shown in Figure 5. Generally, the substituted ester groups, especially the groups on the highly active C6, are firstly decomposed in the process of thermal decomposition [29]. In the step of the decomposition of ester groups, the CAB-MA revealed an initial decomposition temperature and a DTG peak temperature at $189^{\circ} \mathrm{C}$ and $263{ }^{\circ} \mathrm{C}$, respectively, which were lower than that of CAB-LP ( 210 and $284^{\circ} \mathrm{C}$, respectively). These results are in consonance with the above-mentioned ${ }^{13} \mathrm{C}$ NMR analysis, further confirming that MA is efficient technology to activate the cellulose for an esterification reaction with butyric acid and acetic anhydride.

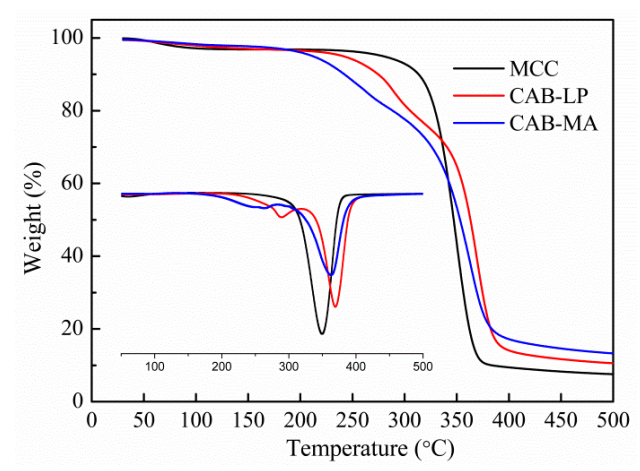

Figure 5. Thermogravimetric analysis (TGA) and derivative thermogravimetry (DTG) curves of MCC and $\mathrm{CAB}$. 


\subsection{Morphology of EB/CAB Microspheres}

The $C A B$, with two kinds of substituted ester groups, presenting the hydrophobicity, is considered to be a favorable drug carrier of hydrophobic drugs such as EB [30]. In this study, EB was successfully entrapped into the $\mathrm{CAB}$ polymer matrix via the solvent evaporation method. The prepared $\mathrm{EB} / \mathrm{CAB}$ microspheres are faint yellow, and present a good dispersion (Figure 6a). The microspheres prepared by both CAB-MA and CAB-LP showed a similar morphology, with numerous pores on their surfaces (Figure $6 \mathrm{~b}, \mathrm{c}$ ). This porous structure would facilitate the infiltration of water to swell or disintegrate the microspheres in the application, which results in the release of the encapsulated drug [31]. Due to a lower molecular weight and a higher DS, CAB-MA had stronger association effect in comparison to CAB-LP, and revealed microspheres with smaller diameters, as determined by the laser particle size distribution instrument (Figure 6d).
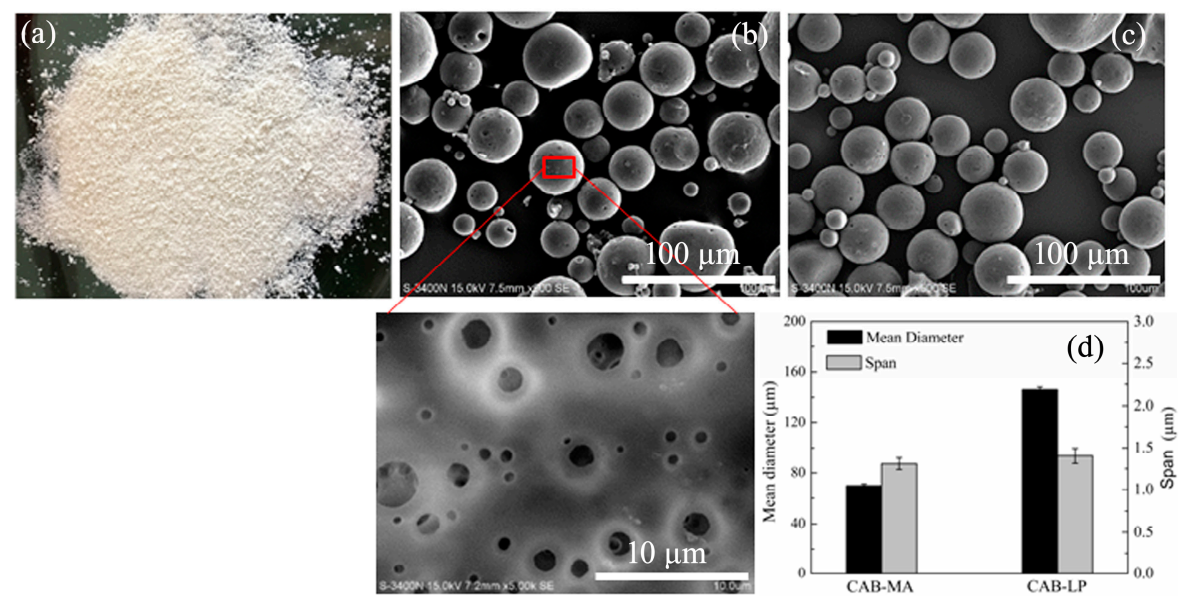

Figure 6. The photograph of the $\mathrm{CAB}$ microspheres containing emamectin benzoate (EB/CAB) (a); the SEM mages of microspheres prepared by CAB-MA (b) and CAB-LP (c), respectively; the mean diameter of EB/CAB-MA and EB/CAB-LP (d).

\subsection{Effect of Synthesis Method on Entrapment Performance of $C A B$}

In order to study the entrapment performance of $C A B s$ prepared by different methods, the $\mathrm{EB} / \mathrm{CAB}-\mathrm{MA}$ and $\mathrm{EB} / \mathrm{CAB}-\mathrm{LP}$ microspheres were produced under the same conditions. The $\mathrm{EB}$ loading (\%), the EB entrapment rate (\%), and the yield of production (\%) for both microspheres are shown in Figure 7. Obviously, these entrapped characteristics of the microcapsules prepared by CAB-MA are greater than that of the microcapsules produced by CAB-LP. Usually, the entrapped characteristics are influenced by the molecular structures of the polymer matrix such as crystallization, molecular weight, and substituted groups [32,33]. The polymer matrix with a lower degree of crystallinity can improve the miscibility between the drug and the matrix, which induces a higher drug loading, entrapped rate, and yield of production in comparison to the polymer with a higher crystallinity. The polymer with a smaller molecular weight and more functional groups that are related to the drug molecular can stabilize the dispersion of a polymer and drug mixture. These also promote the encapsulation performance. Herein, the $\mathrm{CAB}$ prepared by MA technology presented a lower degree of crystallinity, a smaller molecular weight, and a higher content of substituted groups in comparison to the $\mathrm{CAB}$ produced by the LP method. Therefore, the microcapsules derived from CAB-MA exhibited a better encapsulation performance in comparison to the CAB-LP microspheres. 

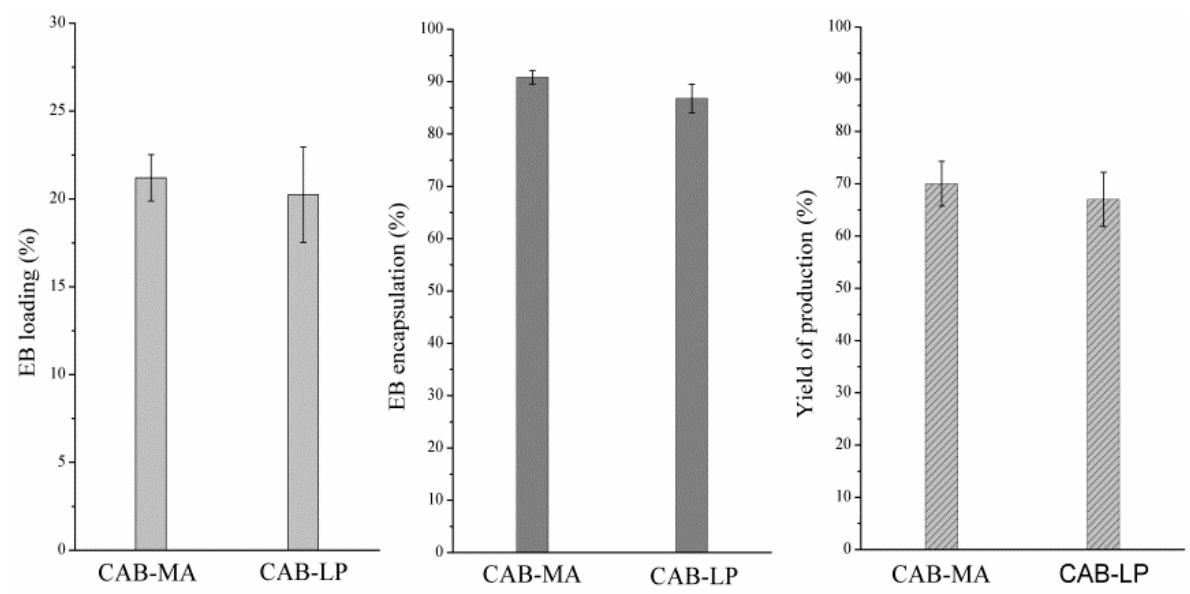

Figure 7. The results of emamectin benzoate (EB) loading, entrapment rate, and yield of production $(n=3)$.

\subsection{EB Release from Microspheres and the Anti-Photodegradation Effect}

Figure 8 shows the release profiles of EB from the EB/CAB-MA microspheres and the EB/CAB-LP microspheres. At the initial stage (10 days), both microspheres displayed a fast release rate of EB, and $30-40 \%$ of EB was released in this period. After that, these microspheres exhibited a slow release rate. At the first step, the EB on the surface or outer layer of the microspheres would be readily discharged, and resulted in a fast release. The EB within the inside of the microspheres was entangled tightly by the $\mathrm{CAB}$ molecular, which prevented the release of $\mathrm{EB}$ until the fracture of the microsphere to produce a new surface. Therefore, the release was slow in this stage. After 20 days, the accumulative release of EB from microspheres was less than $60 \%$. The slow release would prolong the useful life of EB. The structural changes exhibited by the microspheres can be seen in Figure 9. The hydrophilic polyvinyl alcohol, which is a low-content component of EB/CAB microspheres, would be swollen when the microspheres were immersed in the aqueous solution. The swelling of the polyvinyl alcohol would produce a force to rupture the microspheres [34]. Taken together, we could conclude that the release of EB from EB/CAB microspheres followed a self-propelled drug release mechanism [34]. Due to the smaller size, the EB/CAB-MA microspheres with a shorter diffusion path and larger diffusion surface showed a faster release of EB in comparison to the EB/CAB-LP microspheres. To study the kinetics of EB release from the $\mathrm{EB} / \mathrm{CAB}$ microspheres, various kinetic models, including zero-order (Equation (3)), first-order (Equation (4)), and Higuchi square root (Equation (5)) were employed to fit the release results [35].

$$
\begin{gathered}
Q_{\mathrm{t}} / Q_{0}=k t \\
\left(1-Q_{\mathrm{t}} / Q_{0}\right)=k t \\
Q_{\mathrm{t}} / Q_{0}=k t^{0.5}
\end{gathered}
$$

where $Q_{\mathrm{t}}$ and $Q_{0}$ are the amount of EB released at time $t$ and the initial amount of EB in the microspheres, respectively; and $k$ represents the corresponding rate constant. The correlation coefficient $(R)$ values for the fitting were listed in Table 3. With a greater $R$ value, the first-order kinetic model was more consistent with the release of EB from both kinds of microspheres in comparison to the other kinetic models. 


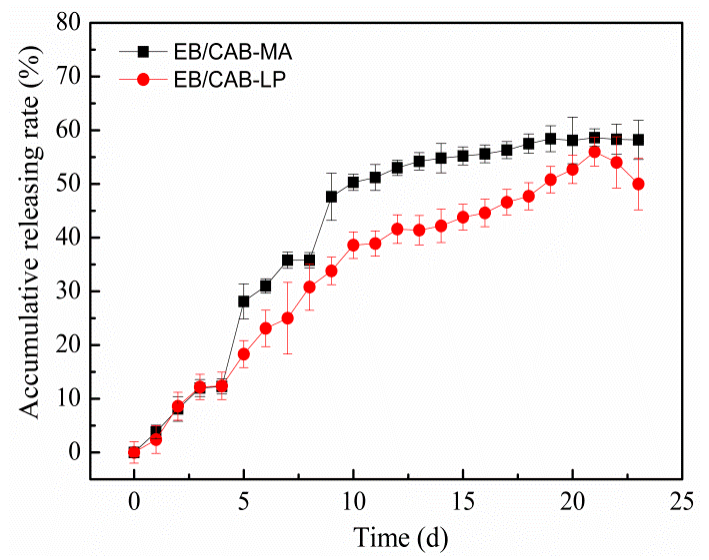

Figure 8. Releasing rate of EB from EB/CAB-MA and EB/CAB-LP microspheres $(n=3)$.

Table 3. $R$ values of all models for releasing EB from EB/CAB-MA microspheres and EB/CAB-LP microspheres.

\begin{tabular}{cccc}
\hline & Zero Order & First Order & Higuchi Equation \\
\hline EB/CAB-MA & 0.8181 & 0.9590 & 0.9132 \\
EB/CAB-LP & 0.9219 & 0.9837 & 0.9633 \\
\hline
\end{tabular}

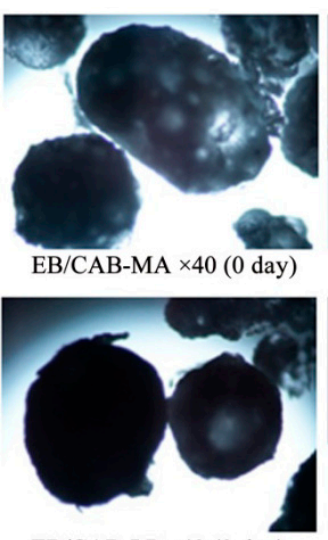

$\mathrm{EB} / \mathrm{CAB}-\mathrm{LP} \times 40$ (0 day)
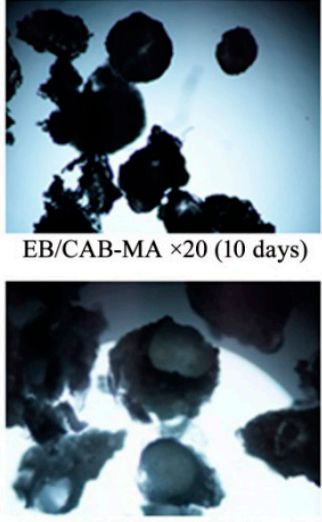

$\mathrm{EB} / \mathrm{CAB}-\mathrm{LP} \times 20$ (20 days)

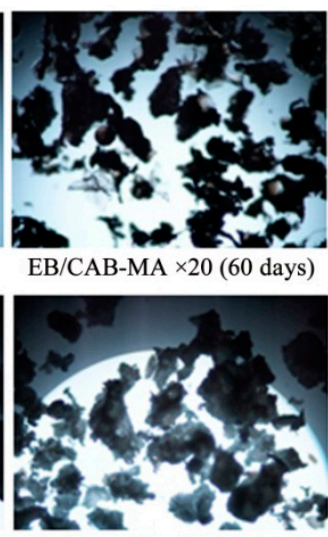

$\mathrm{EB} / \mathrm{CAB}-\mathrm{LP} \times 20(60$ days $)$

Figure 9. The optical photographs of EB/CAB-MA and EB/CAB-LP microspheres during the degradation process.

Figure 10 shows the photodegradation of EB in the control and entrapped in both types of microspheres. After exposure to sunlight for 12 days, more than $80 \%$ of the control EB was degraded. However, the EB that was entrapped in both microspheres exhibited a similar degradation rate, and only about $40 \%$ of the EB was decomposed under the same exposure time. This result indicates that $\mathrm{CAB}$ can encapsulate $\mathrm{EB}$ to protect it from the degradation by sunshine. 


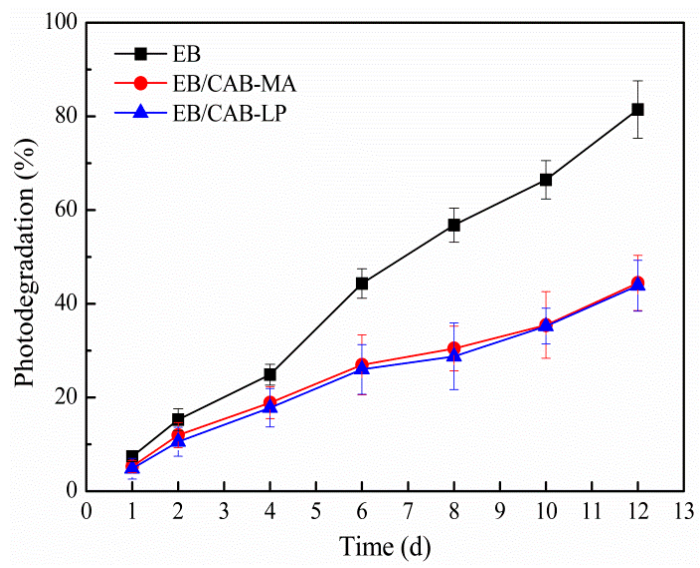

Figure 10. The photodegradation of EB in the control and entrapped in the microspheres $(n=3)$.

\section{Conclusions}

Cellulose acetate butyrate microspheres containing emamectin benzoate were prepared to promote the efficacy of emamectin benzoate by enabling slow release and providing resistance against photolysis. Following the self-propelled release mechanism, less than $60 \%$ of the loading emamectin benzoate was released from the microspheres during the first 20 days. Compared to the cellulose acetate butyrate prepared by the conventional liquid phase method, in which a pretreatment by the mixture of acetic acid and acetic anhydride was needed, the cellulose acetate butyrate produced by the mechanical activation-assisted technology showed a lower crystallinity, a smaller molecular weight, and a higher content of substituted groups. These benefited the pelletization of cellulose acetate butyrate, and improved the combination of polymer molecular and drug molecular, which therefore increased the encapsulation of emamectin benzoate. Moreover, the mechanical activation-assisted technology, without any use of additional solvents, was a highly efficient, easy-handling, and eco-friendly method for producing cellulose acetate butyrate.

Author Contributions: Conceptualization, A.H., Y.Z. and Z.H.; Methodology, A.H.; Investigation, H.H. and X.L. (Xuanhai Li); Writing-Original Draft Preparation, A.H. and X.L. (Xingtang Liang); Writing-Review \& Editing, Y.Z. and Y.Y.; Project Administration, Z.H.

Funding: This work was supported by the National Natural Science Foundation of China $(51463003,21666005$ and 51663020), Guangxi Natural Science Foundation, China (No. 2017GXNSFEA198001, 2018GXNSFAA138037 and 2017GXNSFFA198007), Nanning Scientific Research and Technological Development Project, China (20171109 and 20185066-2), the project of Guangxi Colleges and Universities for the promotion of foundation ability of young teachers (2018KY0599), and Scientific Research Foundation of Guangxi University, China (XJPZ160713).

Conflicts of Interest: The authors declare no conflict of interest.

\section{References}

1. Fanigliulo, A.; Sacchetti, M. Emamectin benzoate: New insecticide against helicoverpa armigera. Commun. Agric. Appl. Biol. Sci. 2008, 73, 651-653. [PubMed]

2. Mushtaq, M.; Chukwudebe, A.C.; Wrzesinski, C.; Allen, L.R.; Luffer-Atlas, D.; Arison, B.H. Photodegradation of emamectin benzoate in aqueous solutions. J. Agric. Food Chem. 1998, 46, 1181-1191. [CrossRef]

3. Singh, B.; Lal, H.; Pal, L.; Sharma, V. In vitro release profile of anti-ulcer drug rabeprazole from biocompatible psyllium-PVA hydrogels. J. Mater. Sci. Mater. Med. 2012, 23, 1021-1032. [CrossRef]

4. Nanaki, S.; Tseklima, M.; Christodoulou, E.; Triantafyllidis, K.; Kostoglou, M.; Bikiaris, D.N. Thiolated chitosan masked polymeric microspheres with incorporated mesocellular silica foam (MCF) for intranasal delivery of paliperidone. Polymers 2017, 9, 617. [CrossRef]

5. Sokolsky-Papkov, M.; Agashi, K.; Olaye, A.; Shakesheff, K.; Domb, A.J. Polymer carriers for drug delivery in tissue engineering. Adv. Drug Deliv. Rev. 2007, 59, 187-206. [CrossRef] [PubMed] 
6. Qi, L.; Ji, G.; Luo, Z.; Xiao, Z.; Yang, Q. Characterization and drug delivery properties of OSA starch-based nanoparticles prepared in $\left[\mathrm{C}_{3} \mathrm{OHmim}\right]$ Ac-in-oil microemulsions system. ACS Sustain. Chem. Eng. 2017, 5, 9517-9526. [CrossRef]

7. Jonoobi, M.; Oladi, R.; Davoudpour, Y.; Oksman, K.; Dufresne, A.; Hamzeh, Y.; Davoodi, R. Different preparation methods and properties of nanostructured cellulose from various natural resources and residues: A review. Cellulose 2015, 22, 935-969. [CrossRef]

8. Arca, H.C.; Mosquera-Giraldo, L.I.; Bi, V.; Xu, D.; Taylor, L.S.; Edgar, K.J. Pharmaceutical applications of cellulose ethers and cellulose ether esters. Biomacromolecules 2018, 19, 2351-2376. [CrossRef]

9. Dewangan, A.K.; Perumal, Y.; Pavurala, N.; Chopra, K.; Mazumder, S. Preparation, characterization and anti-inflammatory effects of curcumin loaded carboxymethyl cellulose acetate butyrate nanoparticles on adjuvant induced arthritis in rats. J. Drug Deliv. Sci. Technol. 2017, 41, 269-279. [CrossRef]

10. Porcu, E.P.; Salis, A.; Rassu, G.; Maestri, M.; Galafassi, J.; Bruni, G.; Giunchedi, P.; Gavini, E. Engineered polymeric microspheres obtained by multi-step method as potential systems for transarterial embolization and intraoperative imaging of HCC: Preliminary evaluation. Eur. J. Pharm. Biopharm. 2017, 117, 160-167. [CrossRef]

11. Mahmood Raouf, R.; Abdul Wahab, Z.; Azowa Ibrahim, N.; Abidin Talib, Z.; Chieng, B.W. Transparent blend of poly(methylmethacrylate)/cellulose acetate butyrate for the protection from ultraviolet. Polymers 2016, 8 , 128. [CrossRef]

12. Fundueanu, G.; Constantin, M.; Esposito, E.; Cortesi, R.; Nastruzzi, C.; Menegatti, E. Cellulose acetate butyrate microcapsules containing dextran ion-exchange resins as self-propelled drug release system. Biomaterials 2005, 26, 4337-4347. [CrossRef] [PubMed]

13. Gan, T.; Zhang, Y.; Chen, Y.; Hu, H.; Yang, M.; Huang, Z.; Chen, D.; Huang, A. Reactivity of main components and substituent distribution in esterified sugarcane bagasse prepared by effective solid phase reaction. Carbohyd. Polym. 2018, 181, 633-641. [CrossRef] [PubMed]

14. Hu, H.; Liu, W.; Shi, J.; Huang, Z.; Zhang, Y.; Huang, A.; Yang, M.; Qin, X.; Shen, F. Structure and functional properties of octenyl succinic anhydride modified starch prepared by a non-conventional technology. Starch-Stärke 2016, 68, 151-159. [CrossRef]

15. Zhao, X.; Huang, Z.; Zhang, Y.; Yang, M.; Chen, D.; Huang, K.; Hu, H.; Huang, A.; Feng, Z. Efficient solid-phase synthesis of acetylated lignin and a comparison of the properties of different modified lignins. J. Appl. Polym. Sci. 2017, 134, 4276. [CrossRef]

16. Zhao, X.; Zhang, Y.; Hu, H.; Huang, Z.; Yang, M.; Chen, D.; Huang, K.; Huang, A.; Qin, X.; Feng, Z. Effect of mechanical activation on structure changes and reactivity in further chemical modification of lignin. Int. J. Biol. Macromol. 2016, 91, 1081-1089. [CrossRef] [PubMed]

17. Zhao, X.; Zhang, Y.; Yang, M.; Huang, Z.; Hu, H.; Huang, A.; Feng, Z. Acylation of lignin with different acylating agents by mechanical activation-assisted solid phase synthesis: Preparation and properties. Polymers 2018, 10, 907. [CrossRef]

18. Zhang, Y.; Wei, L.; Hu, H.; Zhao, Z.; Huang, Z.; Huang, A.; Shen, F.; Liang, J.; Qin, Y. Tribological properties of nano cellulose fatty acid esters as ecofriendly and effective lubricant additives. Cellulose 2018, 25, 3091-3103. [CrossRef]

19. Tezuka, Y.; Tsuchiya, Y. Determination of substituent distribution in cellulose acetate by means of a ${ }^{13} \mathrm{C}$ NMR study on its propanoated derivative. Carbohyd. Res. 1995, 273, 83-91. [CrossRef]

20. Babu, V.R.; Rao, K.K.; Lee, Y.I. Preparation and characterization of nifedipine-loaded cellulose acetate butyrate based microspheres and their controlled release behavior. Polym. Bull. 2010, 65, 157-167. [CrossRef]

21. Hornig, S.; Heinze, T. Efficient approach to design stable water-dispersible nanoparticles of hydrophobic cellulose esters. Biomacromolecules 2008, 9, 1487-1492. [CrossRef]

22. Bert, V.; Wagenknecht, W. Substitution patterns of cellulose ethers influence of the synthetic pathway. Macromol. Symp. 2008, 262, 97-118. [CrossRef]

23. Ispas-Szabo, P.; Ravenelle, F.; Hassan, I.; Preda, M.; Mateescu, M.A. Structure-properties relationship in cross-linked high-amylose starch for use in controlled drug release. Carbohyd. Res. 1999, 323, 163-175. [CrossRef]

24. Lopes, D.G.; Becker, K.; Stehr, M.; Lochmann, D.; Haack, D.; Zimmer, A.; Salar-Behzadi, S. Role of lipid blooming and crystallite size in the performance of highly soluble drug-loaded microcapsules. J. Pharm. Sci. 2015, 104, 4257-4265. [CrossRef] [PubMed] 
25. Yadav, S.K.; Khilar, K.C.; Suresh, A.K. Release rates from semi-crystalline polymer microcapsules formed by interfacial polycondensation. J. Membrane Sci. 1997, 125, 213-218. [CrossRef]

26. Wang, L.; Han, G.; Zhang, Y. Comparative study of composition, structure and properties of Apocynum venetum fibers under different pretreatments. Carbohyd. Polym. 2007, 69, 391-397. [CrossRef]

27. Segal, L.G.J.M.A.; Creely, J.J.; Martin, A.E., Jr.; Conrad, C.M. An empirical method for estimating the degree of crystallinity of native cellulose using the X-ray diffractometer. Text. Res. J. 1959, 29, 786-794. [CrossRef]

28. Lorenzo, G.; Zaritzky, N.; Califano, A. Modeling rheological properties of low-in-fat o/w emulsions stabilized with xanthan/guar mixtures. Food Res. Int. 2008, 41, 487-494. [CrossRef]

29. Elanthikkal, S.; Gopalakrishnapanicker, U.; Varghese, S.; Guthrie, J.T. Cellulose microfibres produced from banana plant wastes: Isolation and characterization. Carbohyd. Polym. 2010, 80, 852-859. [CrossRef]

30. Edgar, K.J. Cellulose esters in drug delivery. Cellulose 2007, 14, 49-64. [CrossRef]

31. Uhrich, K.E.; Cannizzaro, S.M.; Langer, R.S.; Shakesheff, K.M. Polymeric systems for controlled drug release. Chem. Rev. 1999, 99, 3181-3198. [CrossRef] [PubMed]

32. Katona, J.M.; Sovilj, V.J.; Petrović, L.B. Microencapsulation of oil by polymer mixture-ionic surfactant interaction induced coacervation. Carbohyd. Polym. 2010, 79, 563-570. [CrossRef]

33. Petrovic, L.B.; Sovilj, V.J.; Katona, J.M.; Milanovic, J.L. Influence of polymer-surfactant interactions on o/w emulsion properties and microcapsule formation. J. Colloid Interface Sci. 2010, 342, 333-339. [CrossRef] [PubMed]

34. Wu, Y.; Lin, X.; Wu, Z.; Möhwald, H.; He, Q. Self-propelled polymer multilayer Janus capsules for effective drug delivery and light-triggered release. ACS Appl. Mater. Inter. 2014, 6, 10476-10481. [CrossRef] [PubMed]

35. Phadke, K.V.; Manjeshwar, L.S.; Aminabhavi, T.M.; Sathisha, M.P. Cellulose acetate butyrate bilayer coated microspheres for controlled release of ciprofloxacin. Polym. Bull. 2018, 75, 1329-1348. [CrossRef]

(C) 2018 by the authors. Licensee MDPI, Basel, Switzerland. This article is an open access article distributed under the terms and conditions of the Creative Commons Attribution (CC BY) license (http:/ / creativecommons.org/licenses/by/4.0/). 